(1)

CrossMark

\title{
Putative contributions of circadian clock and sleep in the context of SARS-CoV-2 infection
}

\author{
Miguel Meira e Cruz ${ }^{1,4}$, Masaaki Miyazawa ${ }^{2,4}$ and David Gozal (10 ${ }^{3}$
}

Affiliations: 'Sleep Unit, Cardiovascular Center of University of Lisbon, Lisbon School of Medicine, Lisbon, Portugal. 'Dept of Immunology, Faculty of Medicine and Anti-Aging Center, Kindai University, Osaka, Japan. ${ }^{3}$ Dept of Child Health and the Child Health Research Institute, The University of Missouri School of Medicine, Columbia, MO, USA. ${ }^{4}$ Equal contributors.

Correspondence: David Gozal, Dept of Child Health, University of Missouri School of Medicine, 400 N. Keene Street, Columbia, MO 65201, USA. E-mail: gozaldahealth.missouri.edu

@ERSpublications

Circadian deregulation and poor or insufficient sleep may facilitate COVID-19 infection and severity https://bit.ly/2VUlIIJ

Cite this article as: Meira e Cruz M, Miyazawa M, Gozal D. Putative contributions of circadian clock and sleep in the context of SARS-CoV-2 infection. Eur Respir J 2020; 55: 2001023 [https://doi.org/10.1183/ 13993003.01023-2020].

\section{Introduction}

Severe acute respiratory syndrome coronavirus 2 (SARS-CoV-2), the aetiological agent of the pandemic coronavirus disease 2019 (COVID-19), is a newly found member of the Coronaviridae family, and is closely related to, albeit with important differences from, SARS-CoV [1]. It enters human cells through the binding of surface spike (S) glycoprotein with angiotensin-converting enzyme 2 (ACE2) [2-4]. The distal S1 subunit of the S protein is responsible for receptor binding, while the transmembrane S2 subunit mediates fusion between the viral envelope and the target cell membrane following proteolytic cleavage by specific cellular enzymes such as transmembrane serine protease 2 for S protein priming [5]. As it is likely that expression levels of ACE2 affect the efficiency of virus attachment and entry, as well as disease severity [6], and the interactions between viral S protein and ACE2 may directly cause lung injury [7], ACE2 may be a potential target of therapeutic and preventative interventions [8].

\section{Viral infection pathophysiology and the role of the circadian clock system}

The pathogenicity of viral infections can be affected by the host's circadian clock system via two different mechanisms [9-11] (figure 1): 1) direct regulation of viral replication within the target cells; and 2) indirect effects on innate and adaptive immune responses. For example, BMAL1, one of the key regulators of the circadian oscillator, directly affects mouse herpes virus infection in cultured cells and herpes virus replication is significantly enhanced in cells lacking the BMAL1 molecule [13]. Conversely, acute infection with mouse herpes virus increases BMAL1 expression, which consequently deranges or enhances cell-autonomous rhythms depending at what point in the circadian cycle the infection takes place. Absence of BMAL1 affects the expression of cellular factors involved in protein biosynthesis, endoplasmic reticulum function and vesicular trafficking, all of which are important elements in intracellular replication of coronaviruses [14]. Similarly, BMAL1 and REV-ERB $\alpha$, the nuclear receptor family intracellular transcription factor required for synchronising and maintaining the peripheral clock [15], influence 


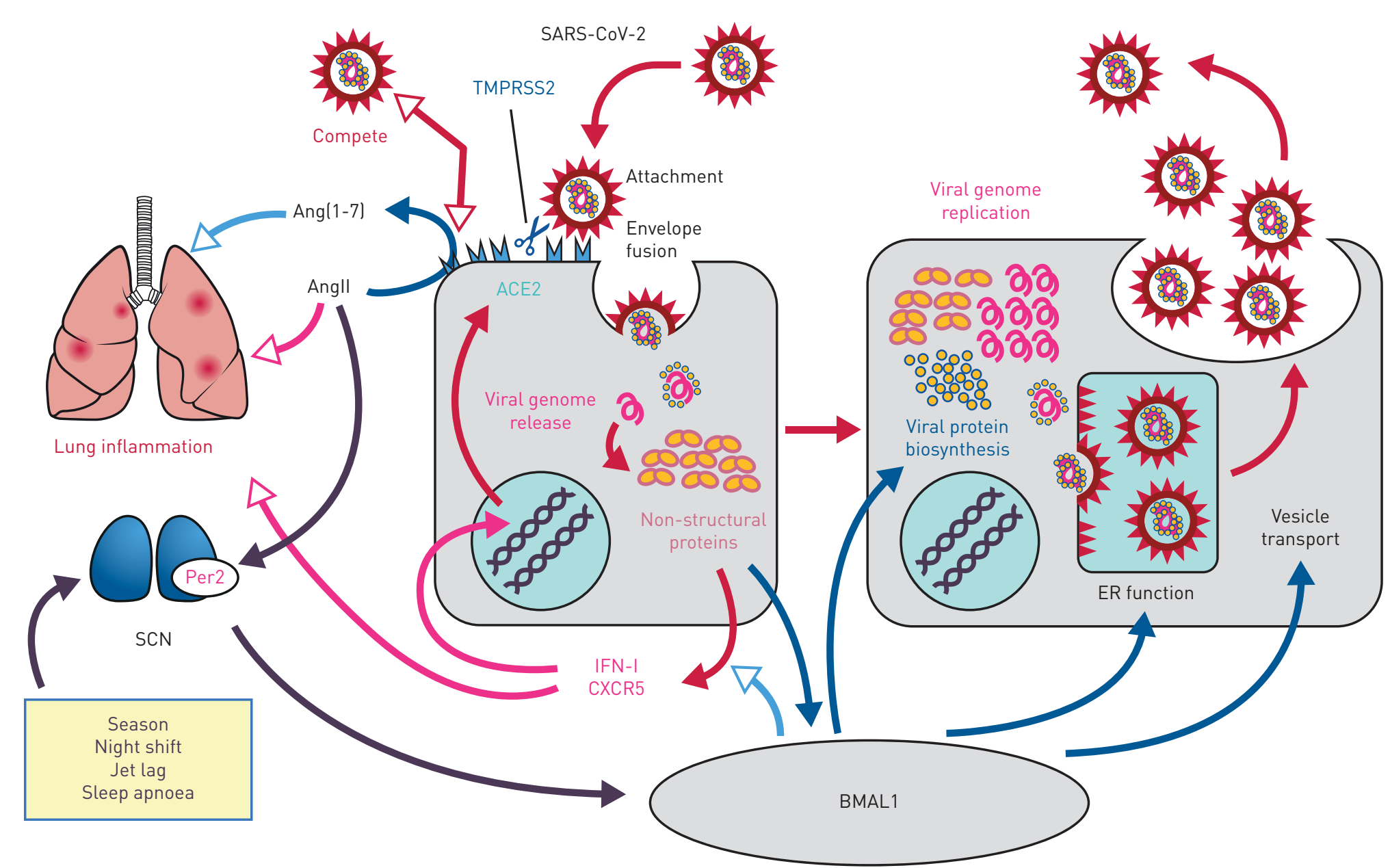

FIGURE 1 Possible links between severe acute respiratory syndrome coronavirus 2 (SARS-CoV-2) infection and the circadian clock system. Blue arrows indicate known links between cellular BMAL1 expression and replication of or responses to other viruses. Angiotensin-converting enzyme 2 (ACE2) expression on respiratory epithelial cells may be regulated through type 1 interferon (IFN-I)-induced mechanisms [12]. As the surface S glycoprotein of SARS-CoV-2 binds the protease domain of ACE2, virus attachment may directly compete with the processing of angiotensin II (ATII) to Ang(1-7), a negative regulator of the renin-angiotensin system. ATII is known to be proinflammatory, while Ang(1-7) is anti-inflammatory. IFN-I signalling in the late phase of coronavirus infection induces exaggerated cytokine production and more severe lung injury. BMAL1 regulates IFN-I and chemokine CXCL5 production. ATII is known to affect the central clock system in the suprachiasmatic nuclei (SCN) through its effect on Per2 levels. TMPRSS2: transmembrane serine protease 2; ER: endoplasmic reticulum. 
multiple steps in the hepatitis $\mathrm{C}$ virus life cycle, including its ability to enter hepatocytes as well as the RNA genome replication of the virus within hepatocytes. Knock-out approaches of Bmal1, genetic over-expression or increased REV-ERB activity using synthetic agonists, markedly reduce the replication of not only hepatitis C virus but also of other flaviviruses, such as Dengue and Zika viruses, through disruption of lipid signalling pathways [16].

It is now well established that there is seasonal variation of BMAL1 expression, with the lowest values being recorded during winter, and these variations have been implicated in the high prevalence of respiratory viral diseases during winter, particularly in light of the observation that low BMAL1 expression enhances viral virulence [13]. When mice were infected with herpes virus at various time-points of the day, significant effects were ascribed to the circadian clock [17]. Thus, the circadian clock appears to influence both the infectivity of viruses as well as their ability to replicate inside the host. Furthermore, we surmise that since the circadian clock machinery is intrinsically involved in the regulation of angiotensin pathways, particularly in the transcriptional expression of ACE2, an imputed role for the circadian clock in regulating the infectivity to SARS-CoV-2 is highly probable [18].

The host circadian clock has now been recognised as a major modulator of immune/inflammatory responses in general [19-21], and in particular following respiratory virus infections in vivo. It has been shown that an endogenous circadian clock within lung epithelial cells modulates neutrophil recruitment through the chemokine ligand CXCL5 [22]. Genetic ablation of BMAL1 in the bronchioles resulted in a disruption of CXCL5 expression rhythms, leading to exaggerated inflammatory responses upon lipopolysaccharide stimulation. Depletion of BMAL1 also resulted in increased morbidity, mortality and virus replication in mice due to a lack of circadian regulation of interferon expression. Importantly, environmental disruption of circadian function by an experimental chronic jet lag model resulted in disrupted temporal expression of BMAL1 in the lungs and increased weight loss after respiratory Sendai virus infection [23].

The time of infection not only changes susceptibility but also predicts survival after influenza virus infection in mice [24]. Mice infected at the beginning of their active phase showed higher mortality than those infected at the onset of the rest phase. These temporal differences were abolished in mice lacking BMAL1 expression. Interestingly, heightened inflammation was observed in mice infected at the onset of the active phase, while the number of natural killer (NK) cells was significantly higher in the group infected just prior to the rest phase, suggesting that circadian regulation of innate immunity plays a major role in these differences. Indeed, depletion of NK cells abolished the time of day differences, indicating that the molecular clock controls host cell responses to influenza virus infection via NK cell functions.

It should be noted, however, that the roles of the host immune/inflammatory responses in the pathogenesis of influenza virus infection markedly differ from those involved in SARS-CoV-2 infection. Influenza virus replicates vigorously soon after infection and causes massive and sometimes dysregulated production of inflammatory cytokines, potentially leading to the so-called cytokine storm, while replication of SARS-CoV-2 is much slower, and development of lung pathological changes coincides with the activation of the host adaptive immune responses, including those of T-helper cell 17 [25].

Nevertheless, it should be emphasised that the timing of the host type 1 interferon (IFN-I) responses determines the outcome of respiratory coronavirus infections. Both in Middle East respiratory syndrome and SARS-CoV infections, early IFN-I signalling is associated with reduced virus replication and mild lung pathology, while delayed IFN-I signalling causes increased infiltration of inflammatory monocytes, heightened proinflammatory cytokine production and fatal pneumonia [26, 27]. These heightened inflammatory responses might be further exaggerated during BMAL1 dysregulation.

\section{Role of ACE2 in SARS-CoV-2 infection}

SARS-CoV-2 exhibits a high affinity to tissue ACE2. Under normal circumstances, ACE2 is responsible for the inactivation of angiotensin II (ATII) and therefore plays, among other functions, an important role in endothelium and cardiovascular homeostasis [28]. Indeed, the ACE-Ang II-AT1R pathway is called the classical renin-angiotensin system (RAS) pathway, and regulates sympathetic nervous system tension, causes vasoconstriction, increases blood pressure, and promotes inflammation, fibrosis and myocardial hypertrophy, while the ACE2-Ang 1-7-Mas proto-oncogene receptor-based axis is viewed as the counter-regulatory RAS pathway, and antagonises the effects of the classical pathway [28], yet also serves as the tissue receptor for SARS-CoV-2. ACE2 is present in several cellular substrates in the body, but is particularly abundant in the vascular endothelium, including the pulmonary vasculature, which may explain the predilection of SARS-CoV-2 to the lung. In this context, several hypotheses have been raised revolving around the overall effect of ACE1 or angiotensin receptor blockers on COVID-19 infections. Putative beneficial results would consist of ACE2 receptor blockade, thereby restricting the viral entry load 
into organs such as the lungs, and modulation of inflammatory responses by these pharmacological agents. However, a potential retrograde feedback mechanism leading to upregulation of ACE2 receptors cannot be excluded. As such, notwithstanding the a priori beneficial effects of ACE1/angiotensin receptor blockers on SARS-CoV infection, we cannot extrapolate them to SARS-CoV-2 causing COVID-19. Furthermore, when conditions such as aging, systemic hypertension and other cardiovascular diseases are present, increased ATII levels lead to lower ACE2 activity and increased inflammation, and could position such patients at increased risk for deterioration of their underlying disease and more severe adverse outcomes due to inactivation of the already reduced ACE2 by SARS-CoV-2 [29].

\section{The putative role of circadian clocks in the pathophysiology of SARS-CoV-2 infection}

As mentioned, SARS-CoV-2 cellular receptor ACE2 is expressed on the outer membranes of tracheal, bronchiolar and lung alveolar epithelial cells, enterocytes in the small intestine, vascular endothelial and smooth muscle cells, and epithelial cells of renal tubules, along with other mucosal tissues [30]. Importantly, ACE2 functions as a negative regulator of the RAS by cleaving ATII at its C-terminus and creating vasodilating angiotensin 1-7 $(\operatorname{Ang}(1-7))$ [7]. A lack of ACE2 is associated with increased lung injury upon acid aspiration, and this detrimental effect was markedly ameliorated in mice genetically lacking the lung epithelial angiotensin II receptor 1a [31]. Further, administration of recombinant ACE2 protected ACE2-deficient as well as wild-type mice from acute lung injury. Thus, ATII functions as proinflammatory mediator while Ang(1-7) exhibits anti-inflammatory functions [6]. As the expression of ACE2 is reduced in aged individuals, its dual function as SARS-CoV-2 entry receptor and as the enzyme that converts proinflammatory ATII into anti-inflammatory Ang(1-7) may account for the less frequent infection with SARS-CoV-2 but more severe disease outcome in older individuals [6].

Possible circadian changes in the expression of ACE2 in the lungs have not been reported until now, but ATII is known to affect the rhythmic expression of Per2, one of the key repressors that constitutes the feedback loop of mammalian clock circuitry in the suprachiasmatic nuclei [32]. Further, levels of ACE expression in the heart show distinct diurnal changes [33] and ATII induces changes in the expression of circadian genes in the vascular cells [34], while changes in BMAL1 expression also affect the expression of angiotensinogen and resting blood pressure in perivascular adipose tissues [35]. Thus, it is conceivable that lung expression of ACE2 will also manifest circadian changes through cell autonomous regulations and through indirect effects of circadian changes in the RAS. Considering that the more severe manifestations of SARS-CoV-2 infection include acute respiratory distress syndrome (ARDS), a condition characterised by enhanced vascular permeability in the lung $[36,37]$, it would be important to also consider the role of the pulmonary endothelium and its cognate expression of ACE2 as a potential target for the virus-induced ARDS $[38,39]$. It is worth noting that a putative adjuvant role for melatonin has been recently proposed for ARDS in the setting of SARS-CoV-2 infection [40]. Furthermore, the higher propensity for COVID-19 to manifest in obese patients and in males, might be also explained by the significant inter-dependent role played by ACE2 in obesity [41-44], and the downstream systemic inflammation induced by this highly prevalent condition [45], and the sexual dimorphism of such effects [46].

\section{Sleep and SARS-CoV-2 infection}

Sleep, a circadian behavioural manifestation of major regulatory homeostatic mechanisms, such as those associated with immunity, has also been shown to interact with host defenses at the molecular and cellular levels. Indeed, extensive literature has indicated the important role of homeostatic sleep in innate and adaptive immunity [47]. Furthermore, there are clear reciprocal dependencies between sleep duration and quality and the immune responses against viral, bacterial, and parasitic pathogens, the latter altering in turn sleep patterns [47]. Thus, it is likely that improved sleep quality and duration in the population may mitigate the propagation and severity of disease induced by SASRS-CoV-2 infection. Interestingly, and potentially relevant to the SARS-CoV-2-ACE2 pathophysiological interdependency, CARHAN et al. [48] observed in a Drosophila model that adult flies lacking ACER (an ACE2 homologue in Drosophila melanogaster) or those treated with peptidase inhibitors, experienced disrupted night-time sleep. We are unaware of any specific studies examining the effects of sleep on SARS-CoV-2 infection. Nonetheless, we should remark that coronaviruses are found with increased frequency in the tonsillar tissues of children suffering from obstructive sleep apnoea [49].

\section{Potential clinical implications}

As mentioned above, it is unclear how disrupted or insufficient sleep may affect SARS-CoV-2 infection rates and the severity of its clinical manifestations. Typical symptoms of acute lung injury include dyspnoea, hypoxaemia and pulmonary oedema, which may deteriorate into SARS. The cell-based pathological mechanisms of such progression involve activation of immune-inflammatory cascades 
resulting in disruption of the alveolar-capillary barrier [39]. This immuno-inflammatory activation is influenced by the circadian clock, and therefore deregulation of circadian rhythms, such as in night shift workers or social jetlag, could play a disease-specific role by altering the susceptibility to infection or modifying the clinical manifestations of COVID-19. It would be of paramount importance to establish the prevalence and severity of SARS-CoV-2 infection in the population, while ensuring that adequate information regarding their sleep habits and circadian activities is appropriately incorporated. Furthermore, since ACE2, which may be regulated by clock activity, was shown to have a protective effect through suppression of apoptosis of pulmonary endothelial cells, it is possible that chronobiological interventions may mitigate the progression of lung injury, if such interventions occur at a very early stage of the infection [50]. Alternatively, in light of clinical studies that show the relevance of chronoadequacy of ACE inhibitor therapy on hypertension [51], it is possible that administration of such agents in the context of SARS-CoV-2 infection may require more precise alignment with the patient's circadian clock status [52], even though the evidence supporting such pharmacological treatment is doubtful at best [53].

Based on the aforementioned considerations, the presence of underlying sleep disorders, such as obstructive sleep apnoea (OSA), could be viewed as a facilitator of SARS-CoV-2 infection, in light of the well-established alterations of the RAS in this highly prevalent disease [54-59]. It will be important to evaluate whether patients with OSA are more likely to be infected, and whether the clinical outcomes among those infected with SARS-CoV-2 are less favourable.

In summary, the unique potential links between SARS-CoV-2, circadian rhythms and sleep have been reviewed, and suggest the possibility that both of these homeostatic processes may significantly modify the susceptibility to infection as well as the overall clinical manifestation of the disease. Therefore, implementation of healthy sleep measures as a protective strategy against infection, and early detection of patients at risk of more severe disease (e.g. night-shift workers or patients with OSA), may enable improved implementation of supportive measures and lead to better outcomes,

Conflict of interest: None declared.

Support statement: D. Gozal is supported by US National Institutes of Health grants HL130984, AG61824, and HL140548. Funding information for this article has been deposited with the Crossref Funder Registry.

\section{References}

1 Zhu N, Zhang D, Wang W, et al. A novel coronavirus from patients with pneumonia in China, 2019. N Engl J Med 2020; 382: 727-733.

2 Wrapp D, Wang N, Corbett KS, et al. Cryo-EM structure of the 2019-nCoV spike in the prefusion conformation. Science 2020; 367: 1260-1263.

3 Yan R, Zhang Y, Li Y, et al. Structural basis for the recognition of SARS-CoV-2 by full-length human ACE2. Science 2020; 367: 1444-1448.

4 Walls AC, Park YJ, Tortorici MA, et al. Structure, function, and antigenicity of the SARS-CoV-2 spike glycoprotein. Cell 2020; 181: 281-292.

5 Hoffmann M, Kleine-Weber H, Schroeder S, et al. SARS-CoV-2 cell entry depends on ACE2 and TMPRSS2 and is blocked by a clinically proven protease inhibitor. Cell 2020; 181: 271-280.

6 AlGhatrif M, Cingolani O, Lakatta EG. The dilemma of coronavirus disease 2019, aging, and cardiovascular disease: insights from cardiovascular aging science. JAMA Cardiol 2020; in press [https://doi.org/10.1001/ jamacardio.2020.1329].

7 Zhang H, Penninger JM, Li Y, et al. Angiotensin-converting enzyme 2 (ACE2) as a SARS-CoV-2 receptor: molecular mechanisms and potential therapeutic target. Intensive Care Med 2020; 46: 586-590.

8 Tai W, He L, Zhang X, et al. Characterization of the receptor-binding domain (RBD) of 2019 novel coronavirus: implication for development of RBD protein as a viral attachment inhibitor and vaccine. Cell Mol Immunol 2020; 19: $1-8$.

9 Zhuang X, Rambhatla SB, Lai AG, et al. Interplay between circadian clock and viral infection. J Mol Med 2017; 95: $1283-1289$.

10 Mazzoccoli G, Vinciguerra M, Carbone A, et al. The circadian clock, the immune system, and viral infections: the intricate relationship between biological time and host-virus interaction. Pathogens 2020; 9: E83.

11 Costantini C, Renga G, Sellitto F, et al. Microbes in the era of circadian medicine. Front Cell Infect Microbiol 2020; 10: 30 .

12 Ziegler C, Allon SJ, Nyquist SK, et al. SARS-CoV-2 receptor ACE2 is an interferon-stimulated gene in human airway epithelial cells and is enriched in specific cell subsets across tissues. Cell 2020; in press [https://doi.org/ 10.2139/ssrn.3555145].

13 Edgar RS, Stangherlin A, Nagy AD, et al. Cell autonomous regulation of herpes and influenza virus infection by the circadian clock. Proc Natl Acad Sci USA 2016; 113: 10085-10090.

14 Fehr AR, Perlman S. Coronaviruses: an overview of their replication and pathogenesis. Methods Mol Biol 2015; 1282: $1-23$.

15 Yin L, Wang J, Klein PS, et al. Nuclear receptor Rev-erbalpha is a critical lithium-sensitive component of the circadian clock. Science 2006; 311: 1002-1005.

16 Zhuang X, Magri A, Hill M, et al. The circadian clock components BMAL1 and REV-ERB $\alpha$ regulate flavivirus replication. Nat Commun 2019; 10: 377. 
17 Matsuzawa T, Nakamura Y, Ogawa Y, et al. Differential day-night outcome to HSV-2 cutaneous infection. J Invest Dermatol 2018; 138: 233-236.

18 Herichova I, Zsoldosova K, Vesela A, et al. Effect of angiotensin II infusion on rhythmic clock gene expression and local renin-angiotensin system in the aorta of Wistar rats. Endocr Regul 2014; 48: 144-151.

19 Murakami M, Tognini P. The circadian clock as an essential molecular link between host physiology and microorganisms. Front Cell Infect Microbiol 2019; 9: 469.

20 Tognini P, Thaiss CA, Elinav E, et al. Circadian coordination of antimicrobial responses. Cell Host Microbe 2017; 22: 185-192.

21 Nguyen KD, Fentress SJ, Qiu Y, et al. Circadian gene bmall regulates diurnal oscillations of ly6c(hi) inflammatory monocytes. Science 2013; 341: 1483-1488.

22 Pick R, He W, Chen CS, et al. Time-of-day-dependent trafficking and function of leukocyte subsets. Trends Immunol 2019; 40: 524-537.

23 Arjona A, Sarkar DK. Evidence supporting a circadian control of natural killer cell function. Brain Behav Immun 2006; 20: 469-476.

24 Gibbs J, Ince L, Matthews L, et al. An epithelial circadian clock controls pulmonary inflammation and glucocorticoid action. Nat Med 2014; 20: 919-926.

25 Ehlers A, Xie W, Agapov E, et al. BMAL1 links the circadian clock to viral airway pathology and asthma phenotypes. Mucosal Immunol 2018; 11: 97-111.

26 Channappanavar R, Fehr AR, Zheng J, et al. IFN-I response timing relative to virus replication determines MERS coronavirus infection outcomes. J Clin Invest 2019; 130: 3625-3639.

27 Channappanavar R, Fehr AR, Vijay R, et al. Dysregulated type I interferon and inflammatory monocyte-macrophage responses cause lethal pneumonia in SARS-CoV-infected mice. Cell Host Microbe 2016; 19: 181-193.

28 Ocaranza M P, Riquelme JA, García L, et al. Counter-regulatory renin-angiotensin system in cardiovascular disease. Nat Rev Cardiol 2020; 17: 116-129.

29 Liu PP, Blet A, Smyth D, et al. The science underlying COVID-19: implications for the cardiovascular system. Circulation 2020; in press [https://doi.org/10.1161/CIRCULATIONAHA.120.047549].

30 Sengupta S, Tang SY, Devine JC, et al. Circadian control of lung inflammation in influenza infection. Nat Commun 2019; 10: 4107.

31 Xu Z, Shi L, Wang Y, et al. Pathological findings of COVID-19 associated with acute respiratory distress syndrome. Lancet Respir Med 2020; 8: 420-422.

$32 \mathrm{Li} \mathrm{W}$, Zhang C, Sui J, et al. Receptor and viral determinants of SARS-coronavirus adaptation to human ACE2. EMBO J 2005; 24: 1634-1643.

33 Imai Y, Kuba K, Rao S, et al. Angiotensin-converting enzyme 2 protects from severe acute lung failure. Nature 2005; 436: 112-116.

34 Naito $\mathrm{Y}$, Tsujino T, Matsumoto $\mathrm{M}$, et al. The mechanism of distinct diurnal variations of renin-angiotensin system in aorta and heart of spontaneously hypertensive rats. Clin Exp Hypertens 2009; 31: 625-638.

35 Nonaka H, Emoto N, Ikeda K, et al. Angiotensin II induces circadian gene expression of clock genes in cultured vascular smooth muscle cells. Circulation 2001; 104: 1746-1748.

36 Chang L, Xiong W, Zhao X, et al. Bmall in perivascular adipose tissue regulates resting-phase blood pressure through transcriptional regulation of angiotensinogen. Circulation 2018; 138: 67-79.

37 Villar J, Zhang H, Slutsky AS. Lung repair and regeneration in ARDS: role of PECAM1 and Wnt signaling. Chest 2019; 155: 587-594.

38 Wang T, Gross C, Desai AA, et al. Endothelial cell signaling and ventilator-induced lung injury: molecular mechanisms, genomic analyses, and therapeutic targets. Am J Physiol Lung Cell Mol Physiol 2017; 312: L452-L476.

39 Fedson DS, Opal SM, Rordam OM. Hiding in plain sight: an approach to treating patients with severe COVID-19 infection. mBio 2020; 11: e00398-20.

40 Kim J, Choi SM, Lee J, et al. Effect of renin-angiotensin system blockage in patients with acute respiratory distress syndrome: a retrospective case control study. Korean J Crit Care Med 2017; 32: 154-163.

41 Slamkova M, Zorad S, Krskova K. Alternative renin-angiotensin system pathways in adipose tissue and their role in the pathogenesis of obesity. Endocr Regul 2016; 50: 229-240.

42 Than A, Leow MK, Chen P. Control of adipogenesis by the autocrine interplays between angiotensin 1-7/Mas receptor and angiotensin II/AT1 receptor signaling pathways. J Biol Chem 2013; 288: 15520-15531.

43 Pinheiro TA, Barcala-Jorge AS, Andrade JMO, et al. Obesity and malnutrition similarly alter the renin-angiotensin system and inflammation in mice and human adipose. J Nutr Biochem 2017; 48: 74-82.

44 Santos SH, Andrade JM. Angiotensin 1-7: a peptide for preventing and treating metabolic syndrome. Peptides 2014; 59: 34-41.

45 Lelis DF, Freitas DF, Machado AS, et al. Angiotensin-(1-7), adipokines and inflammation. Metab Clin Exp 2019; 95: 36-45.

46 Gupte M, Thatcher SE, Boustany-Kari CM, et al. Angiotensin converting enzyme 2 contributes to sex differences in the development of obesity hypertension in C57BL/6 mice. Arterioscler Thromb Vasc Biol 2012; 32: 1392-1399.

47 Zhang R, Wang X, Ni L, et al. COVID-19: melatonin as a potential adjuvant treatment. Life Sci 2020; 250: 117583.

48 Carhan A, Tang K, Shirras CA, et al. Loss of angiotensin-converting enzyme-related (ACER) peptidase disrupts night-time sleep in adult Drosophila melanogaster. J Exp Biol 2011; 214: 680-686.

49 Irwin MR, Opp MR. Sleep health: reciprocal regulation of sleep and innate immunity. Neuropsychopharmacology 2017; 42: 129-155.

50 Yeshuroon-Koffler K, Shemer-Avni Y, Keren-Naus A, et al. Detection of common respiratory viruses in tonsillar tissue of children with obstructive sleep apnea. Pediatr Pulmonol 2015; 50: 187-195.

51 Bao H, Gao F, Xie G, et al. Angiotensin-converting enzyme 2 inhibits apoptosis of pulmonary endothelial cells during acute lung injury through supressing MIR-4262. Cell Physiol Biochem 2015; 37: 759-767.

52 Hermida R, Ayala D. Chronotherapy with the angiotensine-converting enzyme inhibitor ramipril in essential hypertension. Improved blood pressure control with bedtime dosing. Hypertension 2009; 54: 40-46.

53 Rico-Mesa JS, White A, Anderson AS. Outcomes in patients with COVID-19 infection taking ACEI/ARB. Curr Cardiol Rep 2020; 22: 31. 
54 Braun R, Kath WL, Iwanaszko $\mathrm{M}$, et al. Universal method for robust detection of circadian state from gene expression. Proc Natl Acad Sci USA 2018; 115: E9247-E9256.

55 Nicholl DDM, Hanly PJ, Zalucky AA, et al. CPAP therapy delays cardiovagal reactivation and decreases arterial renin-angiotensin system activity in humans with obstructive sleep apnea. J Clin Sleep Med 2018; 14: 1509-1520.

56 Wang W, Song A, Zeng Y, et al. Telmisartan protects chronic intermittent hypoxic mice via modulating cardiac renin-angiotensin system activity. BMC Cardiovasc Disord 2018; 18: 133.

57 Khayat RN, Varadharaj S, Porter K, et al. Angiotensin receptor expression and vascular endothelial dysfunction in obstructive sleep apnea. Am J Hypertens 2018; 31: 355-361.

58 Jin ZN, Wei YX. Meta-analysis of effects of obstructive sleep apnea on the renin-angiotensin-aldosterone system. J Geriatr Cardiol 2016; 13: 333-343.

59 Benjafield AV, Ayas NT, Eastwood PR, et al. Estimation of the global prevalence and burden of obstructive sleep apnoea: a literature-based analysis. Lancet Respir Med 2019; 7: 687-698. 